University of South Carolina

Scholar Commons

$5-1-2004$

\title{
Control Strategies for Active Power Sharing in a Fuel-Cell-Powered Battery-Charging Station
}

Zhenhua Jiang

University of Miami, zjiang1@miami.edu

Roger A. Dougal

University of South Carolina - Columbia, dougal@engr.sc.edu

Follow this and additional works at: https://scholarcommons.sc.edu/elct_facpub

Part of the Electrical and Computer Engineering Commons

\section{Publication Info}

Published in IEEE Transactions on Industry Applications, Volume 40, Issue 3, 2004, pages 917-924.

http://ieeexplore.ieee.org/xpl/Recentlssue.jsp?punumber=28

(C) 2004 by IEEE

This Conference Proceeding is brought to you by the Electrical Engineering, Department of at Scholar Commons. It has been accepted for inclusion in Faculty Publications by an authorized administrator of Scholar Commons. For more information, please contact digres@mailbox.sc.edu. 


\title{
Control Strategies for Active Power Sharing in a Fuel-Cell-Powered Battery-Charging Station
}

\author{
Zhenhua Jiang, Member, IEEE, and Roger A. Dougal, Senior Member, IEEE
}

\begin{abstract}
This paper presents an effective system design for a fuel-cell-powered battery-charging station and three control strategies for active power sharing among the batteries. This battery-charging station allows multiple batteries to be simultaneously charged. Three control strategies were investigated to coordinate the active power distribution among the battery-charging branches. The baseline control strategy was equal rate charging. Two advanced control strategies, proportional rate charging and pulse current charging, were compared to the baseline strategy. These control strategies were realized in MATLAB/Simulink, and the current and voltage regulations were implemented using the classical proportional-integral control approach. The system simulation was conducted in the Virtual Test Bed by embedding Simulink objects of the controller and co-simulating with MATLAB. The experimental tests were performed by compiling Simulink codes of the controller and downloading to the dSPACE platform to control real hardware. The simulation and experimental results are given. Experimental tests validate these control strategies.
\end{abstract}

Index Terms-Active power sharing, battery, charging station, control strategy, dSPACE, fuel cell, Simulink, Virtual Test Bed (VTB).

\section{INTRODUCTION}

$\mathbf{R}$ ECHARGEABLE batteries, such as lithium-ion cells, are playing an increasingly significant role in the utilization of portable electronic devices such as portable computers, cellular phones, camcorders, etc [1]. Their limited usable time makes it necessary to develop some kind of portable battery-charging system. The field application of a portable charging system may be far away from the utility power systems. In this case, the fuel cell, which is emerging as one of the most promising technologies for the future power sources [2], [3], may provide a good solution for powering the portable battery-charging station [4], [5]. However, the limited power supply of the fuel cell and the nonlinearity of both fuel cells and lithium-ion batteries [6]-[9] present difficulties for the system design. Power converters are,

Paper MSDAD-A 04-01, presented at the 2003 IEEE Applied Power Electronics Conference and Exposition, Miami Beach, FL, February 9-13, and approved for publication in the IEEE TRANSACTIONS ON INDUSTRY APPLICATIONS by the Industrial Automation and Control Committee of the IEEE Industry Applications Society. Manuscript submitted for review February 10, 2003 and released for publication February 17, 2004. This work was supported in part by the U.S. Army Communications and Electronics Comand and the U.S. National Reconnaissance Office under Contract NRO 000-01-C-1034, and by the U.S. Office of Naval Research under Contract N00014-00-1-0368 and Contract N00014-00-1-0131.

The authors are with the Department of Electrical Engineering, University of South Carolina, Columbia, SC 29208 USA (e-mail: jiang@engr.sc.edu; dougal@engr.sc.edu).

Digital Object Identifier 10.1109/TIA.2004.827467 thus, needed in this fuel-cell/battery system and should be controlled properly for the best system efficiency.

In general, the battery-charging station should allow multiple batteries to be simultaneously charged. In order to meet the simultaneous requirements of multiple users, power converters are connected in parallel, each for one battery pack. The initial states of the batteries may be different. A battery with a lower initial state-of-charge may require a larger charging current or otherwise a longer charging time. Furthermore, when charging advanced technology batteries such as lithium-ion cells, it is hazardous to exceed some certain current or voltage limits. Therefore, the power from the fuel-cell power source should be distributed efficiently among these charging branches and the power converters should be regulated appropriately, whereas, the power distribution from a nonlinear and current-limited power source presents some challenges for control algorithm design. Obvious difficulty also arises from the controller design of the power converter because the source and load of the power converter are strongly nonlinear and dynamic.

This paper presents a fuel-cell-powered battery-charging station and control strategies for active power sharing among the batteries. The remainder of this paper is organized as follows. The system architecture of the fuel-cell-powered battery-charging station is proposed in Section II. Section III describes three control strategies that were investigated to coordinate the power distribution among the battery-charging branches. The baseline control strategy was equal rate charging. Two advanced control strategies, proportional rate charging and pulse current charging, were compared to this baseline strategy. Section IV presents the implementation of the charge controllers, which were designed, based on these control strategies, in MATLAB/Simulink for both system simulation and experimental tests. The system simulation was conducted in the Virtual Test Bed (VTB) by embedding Simulink objects of the controller and co-simulating with MATLAB. The simulation results are given in Section V. The experimental tests were performed by compiling Simulink codes of the controller and downloading onto a dSPACE platform to control real hardware. Section VI demonstrates the experiment results and validates the control strategies. Conclusions are made in Section VII.

\section{SySTEM ARChITECTURE DESIGN}

Since the battery-charging station is configured for multiple batteries to be simultaneously charged, many individual charging channels can be built in the charging station. Here, we consider three charging channels, which can represent the 


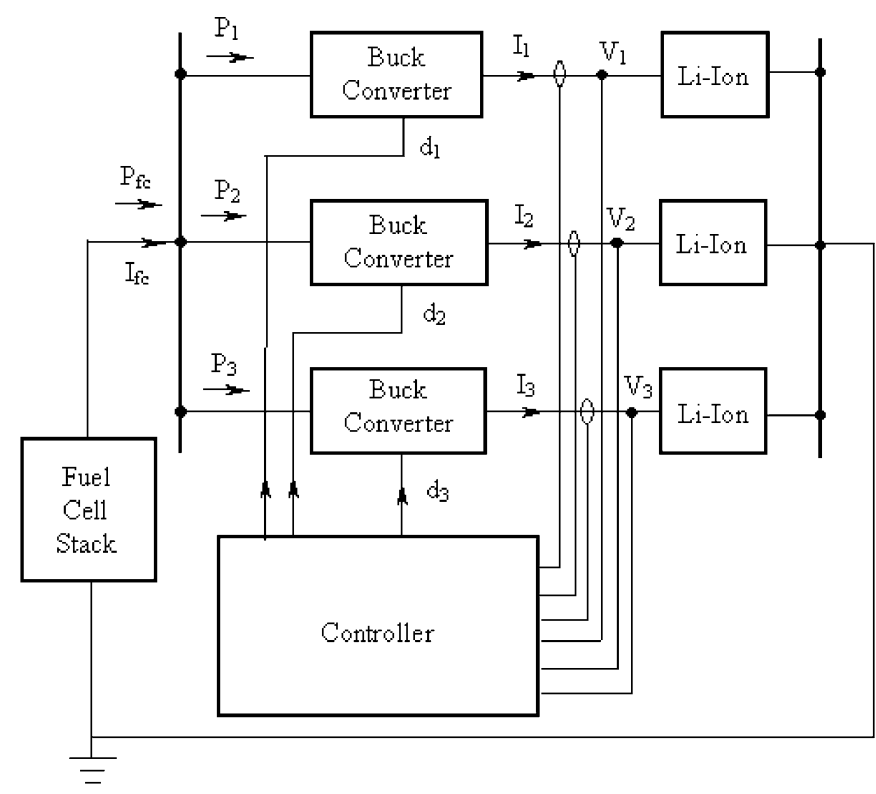

Fig. 1. Block diagram of the system architecture.

general solution of many charging channels. Assume here that all the batteries are put in the charger. The case that some batteries are inserted or retrieved during the charging process is investigated in [10].

The block diagram of the fuel-cell-powered battery-charging station is shown in Fig. 1. A fuel-cell stack, which is the power generation system, is used to charge up to three lithium-ion battery packs through three separate buck converters. Each battery contains four series-connected cells. The buck converters control the charging current and voltage supplied to each battery, and allocate the available power among the batteries according to one of several control strategies that are described here.

As shown in Fig. 1, three buck converters are connected in parallel to a single fuel-cell power source. The power available from the fuel-cell stack is distributed among the three batteries, according to (1)

$$
P_{f c}=P_{1}+P_{2}+P_{3}
$$

where $P_{1}, P_{2}$, and $P_{3}$ are the power to three charging channels, respectively, and $P_{f c}$ is the power from the fuel-cell stack.

In practice, the power distribution among the batteries is realized by regulating the charging currents of the batteries. The following equation relates the current from the fuel-cell stack to three charging currents, assuming no power loss in these power converters:

$$
I_{f c}=d_{1} I_{1}+d_{2} I_{2}+d_{3} I_{3}
$$

where $I_{1}, I_{2}$, and $I_{3}$ are the charging currents to three batteries, respectively, $I_{f c}$ is the current from the fuel-cell stack, and $d_{1}$, $d_{2}$, and $d_{3}$ are the duty cycles of three buck converters, respectively, and they have values between $0-1$.

The sum of the right-hand side in (2) should be less than the maximum current available from the fuel-cell stack. Considering that variations in the voltages of the fuel-cell stack and the batteries are not large, the duty cycle will vary within a limited small range, which means, for instance, values of $d_{1}, d_{2}$, and $d_{3}$ are between $0.70-0.75$. Based on this assumption, we can take the following expression as a criterion for power distribution among the batteries:

$$
I_{1}+I_{2}+I_{3} \leq I_{\lim }
$$

where $I_{\text {lim }}$ is a preset limit for the total charging current that can be estimated according to the maximum current available from the fuel-cell stack and the average duty cycle of power converters. Equation (3) gives a basic requirement for the design of control strategies for active power sharing.

\section{Control Strategies For ACTIVE POWER SHARING}

Different users may have different requirements for charging their batteries. Some people may require that the batteries be fully charged within the shortest period of time, while others wish to maximize the cycle life of their batteries, or minimize the fuel consumption during the charging process. This paper aims to discover the appropriate control schemes for minimizing the total charging time. Three control strategies were investigated to coordinate the power distribution among the battery branches and they are equal rate charging, proportional rate charging, and pulse current charging. The first one is the baseline control strategy, and the other two are compared with it.

Among these control strategies for active power sharing, two kinds of charging protocols may be used. They are constant current-constant voltage (CC-CV) charging protocol and pulse current charging protocol. CC-CV charging protocol can help to protect the battery from overcharging. Under this protocol, the battery is charged to an end potential using a constant current. The potential is then held constant after this potential is reached, and the charging current gradually decreases. The charging process terminates when the current reaches a preset small value during the constant voltage mode. Under pulse current charging protocol, a pulse current with a period of $T$ and pulse duration of $T_{\text {on }}$ is applied to the battery. Pulse current charging protocol has been shown to enhance charging rate capability and to prevent the increase of internal impedance of the battery, thus reducing the total charging time [9].

A straightforward control strategy for charging all of the batteries simultaneously using constant currents is to equally distribute the charging current among them. Due to differences in the initial states of the batteries, some batteries may reach the reference voltage earlier than the others may. When one battery reaches the voltage limit, its voltage will be held constant and its charging current will taper. The rest of the total available current will be distributed equally between the other two batteries. Then, the same scheme is followed for the remaining batteries until all of the batteries are held in the constant voltage mode. This strategy, called equal rate charging here for convenience, is the baseline control strategy for active power sharing. This control strategy is illustrated in Fig. 2(a), and it can be easily implemented on the hardware. The charging time will be as long as it takes to charge the most depleted battery, and, in particular, it may be much longer than the charging time for the less depleted battery to become fully charged when the initial states of charge of the batteries are widely disparate. 


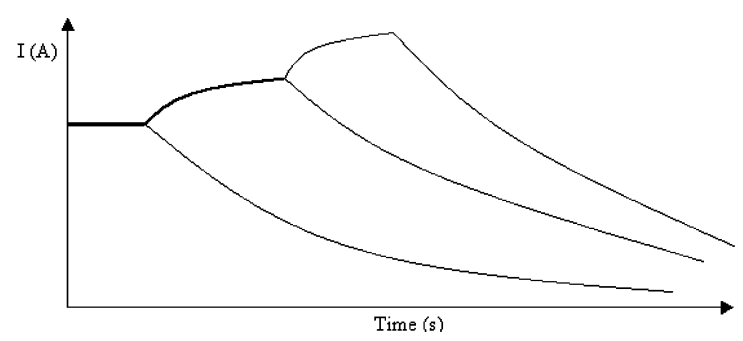

(a)

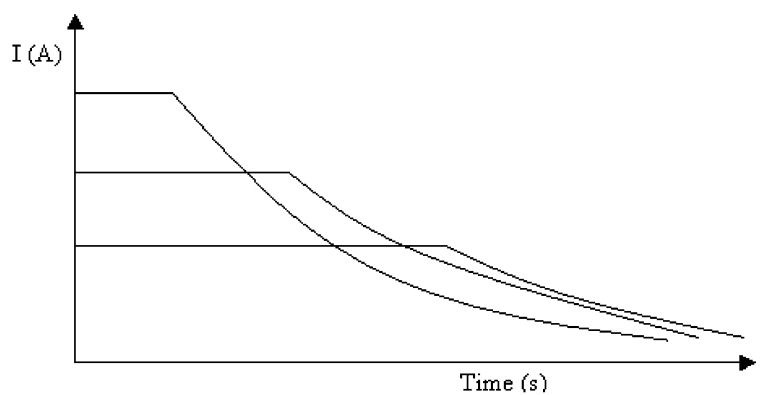

(b)

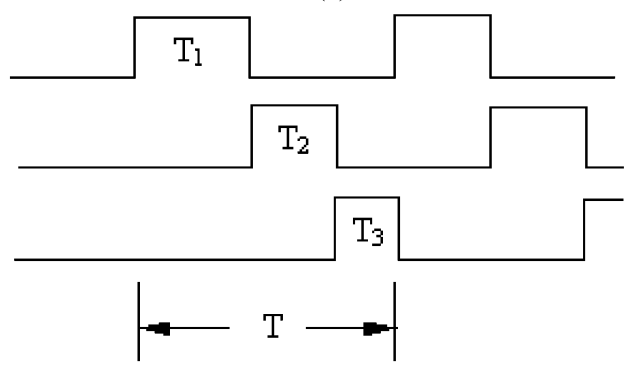

(c)

Fig. 2. Illustration of three control strategies for active power sharing. (a) Equal rate charging. (b) Proportional rate charging. (c) Pulse current charging.

A more time-efficient control strategy can take into account the fact that the charge that any battery will need to become fully charged is proportional to its depth-of-discharge where the depth-of-discharge is defined as unity minus the state-of-charge of this battery. As implied in the previous control strategy, if continuous currents of the same magnitude are applied to charge different batteries, the charging times will be approximately proportional to the depth-of-discharge (neglecting nonlinearity of the batteries). On the other hand, if we want all the batteries to become fully charged during the same period of time, the charging current of each battery can be made proportional to the fraction of the depth-of-discharge of this battery, which can be calculated according to (4)

$$
I_{i}=I_{\lim } \cdot \frac{1-S O C_{i}}{\sum_{i=1}^{3}\left(1-S O C_{i}\right)}, \quad i=1,2,3
$$

where $I_{i}$ is the charging current of the $i$ th battery, $I_{\text {lim }}$ is the total available charging current, and $S O C_{i}$ is the initial state-ofcharge of the $i$ th battery.

This control strategy is called proportional rate charging here, which is shown in Fig. 2(b). Under this strategy, the batteries may become fully charged almost simultaneously. However, it is difficult to estimate the state-of-charge. For lithium-ion batteries, an approximate relationship between the state-of-charge and open-circuit voltage can be found when the state-of-charge is not within the extreme range, i.e., if the state-of-charge is between $0.1-0.9$. When the state-of-charge is greater than 0.9 , the battery charger is usually working under constant voltage mode. The charging current is not determined by the charge controller itself, but by the internal potential and terminal voltage of the battery. It is not necessary to estimate the state-of-charge since the information about state-of-charge is useful only when the charger works under current regulation mode. When the state-of-charge is less than 0.1 , the estimation is cut off to 0.1 . In this case, the estimated value for depth-of-discharge is 0.9 , and the fraction of the depth-of-discharge is very close to that using a more accurate value of the state-of- charge. Therefore, the initial state-of-charge can be estimated by measuring the battery open-circuit voltage and linearly fitting the open-circuit voltage to the state-of-charge. As we will show, even this simple approximation is sufficient for defining the current sharing.

In addition to dc charging, the third control strategy is pulse current charging which uses the pulse current charging protocol while the previous two control strategies use CC-CV charging protocol. With this strategy, three pulse currents with the same period of $T$ and different pulse durations are applied to three batteries alternatively. The sum of the pulse durations of the three pulse currents is equal to the period. This control strategy is illustrated in Fig. 2(c). A strategy similar to proportional rate charging can be defined for pulse current charging by making the duty cycle of each pulse current proportional to the fraction of the depth-of-discharge of this battery. This duty cycle can be estimated according to the following equation:

$$
D_{i}=\frac{1-S O C_{i}}{\sum_{i=1}^{3}\left(1-S O C_{i}\right)}, \quad i=1,2,3
$$

where $D_{i}$ is the duty cycle of the charging current of the $i$ th battery.

Under this strategy, the charging current can be relatively large because only one battery draws this current at any time. Therefore, it can be the limiting current available from the fuel cell as long as it does not exceed the current limit of any battery. With this strategy, it is also possible for all the batteries to become fully charged almost simultaneously. Nevertheless, the disadvantage is that the control algorithm to implement this strategy on the hardware is more complicated compared to dc charging because the output currents of three power converters change frequently resulting in frequent changes in the voltages of the batteries, an undesired fluctuation in the output voltage of the fuel cell, and high dynamics of the system.

The detail of implementing the three control strategies as shown in Fig. 2 is explained as follows. For the convenience of demonstration, assume that initially $V_{1}>V_{2}>V_{3}$ where $V_{1}, V_{2}$, and $V_{3}$ are the voltages of three batteries, respectively. In these strategies, $I_{\text {lim }}$ is the limit of the total charging current; $V_{\text {ref }}$ is battery end potential, which is usually $4.2 \mathrm{~V}$ for each cell; and $I_{1}, I_{2}$, and $I_{3}$ are the charging currents of three batteries, respectively. (Here, we assume that the maximum current available from the fuel cell, $I_{\text {lim }}$, is less than the sum of the maximum safe charging currents for all of the batteries.) 


\section{A. Strategy 1: Equal Rate Charging (Baseline)}

- If $V_{1}<V_{\text {ref }}, I_{\text {ref }, 1}=I_{\text {ref }, 2}=I_{\text {ref }, 3}=I_{\text {lim }} / 3$.

- If $V_{1}=V_{\text {ref }}, I_{1}$ is tapering, and $I_{\text {ref }, 2}=I_{\text {ref }, 3}=\left(I_{\text {lim }}-\right.$ $\left.I_{1}\right) / 2$.

- If $V_{2}=V_{\text {ref }}, I_{2}$ is tapering, and $I_{\text {ref, } 3}=I_{\text {lim }}-I_{1}-I_{2}$.

- If $V_{3}=V_{\text {ref }}, I_{3}$ is tapering.

- If $I_{i}<0.1 \times I_{\mathrm{lim}} / 3$, then $I_{\mathrm{ref}, i}=0$, where $i=1,2,3$.

\section{B. Strategy 2: Proportional Rate Charging}

- $I_{\mathrm{ref}, i}=I_{\lim } *\left(1-S O C_{i}\right) /\left[\left(1-S O C_{1}\right)+\left(1-S O C_{2}\right)+(1-\right.$ $\left.\left.S O C_{3}\right)\right], i=1,2,3$.

- If $V_{1}=V_{\text {ref }}, I_{1}$ is tapering.

- If $V_{2}=V_{\text {ref }}, I_{2}$ is tapering

- If $V_{3}=V_{\text {ref }}, I_{3}$ is tapering.

- If $I_{i}<0.1 \times I_{\text {ref }, i}$, then $I_{\text {ref }, i}=0$, where $i=1,2,3$.

\section{Strategy 3: Pulse Current Charging}

- $I_{\text {high,ref }}=I_{\text {lim }}$, and $I_{\text {low,ref }}=I_{\text {lim }} \times 1 \%$.

- $D_{\text {ref, } i}=\left(1-S O C_{i}\right) /\left[\left(1-S O C_{1}\right)+\left(1-S O C_{2}\right)+(1-\right.$ $\left.\left.S O C_{3}\right)\right]$.

- If $V_{\text {low, } i}>=V_{\text {ref }}$, then $I_{\text {ref, } i}=0$, where $i=1,2,3$.

\section{Synthesis AND IMPLEMENTATION OF CHARGE CONTROLLER}

The charging controllers were designed and implemented in MATLAB/Simulink, which was convenient both for system simulation and for experimental tests. The system simulations were conducted in VTB by embedding Simulink object of the controllers and cosimulating interactively with MATLAB. The experimental tests were performed by compiling Simulink codes of the controllers and downloading onto the dSPACE platform.

The Simulink models of the various charge controllers for the fuel-cell-powered battery-charging station are shown in Fig. 3 for dc charging [Fig. 3(a)] and pulse current charging [Fig. 3(b)], respectively. Both equal rate charging strategy and proportional rate charging strategy can be implemented in the controller shown in Fig. 3(a). Pulse current charging strategy is implemented in the controller shown in Fig. 3(b). The main functional blocks in the controllers are the charging current strategy module, current regulation module, and charging termination decision module. There is a voltage regulation module in the controller shown in Fig. 3(a). The charging current strategy module is to calculate the reference charging currents according to the measured battery voltages and charging currents and it is unique for each of the three different control strategies. The current and voltage regulation modules are used to compute the duty cycles of the buck converters according to the reference currents from the charging current strategy module and the reference voltages, respectively. The charging termination decision module can determine when the charging process stops and output a switching signal to the corresponding charging channel.

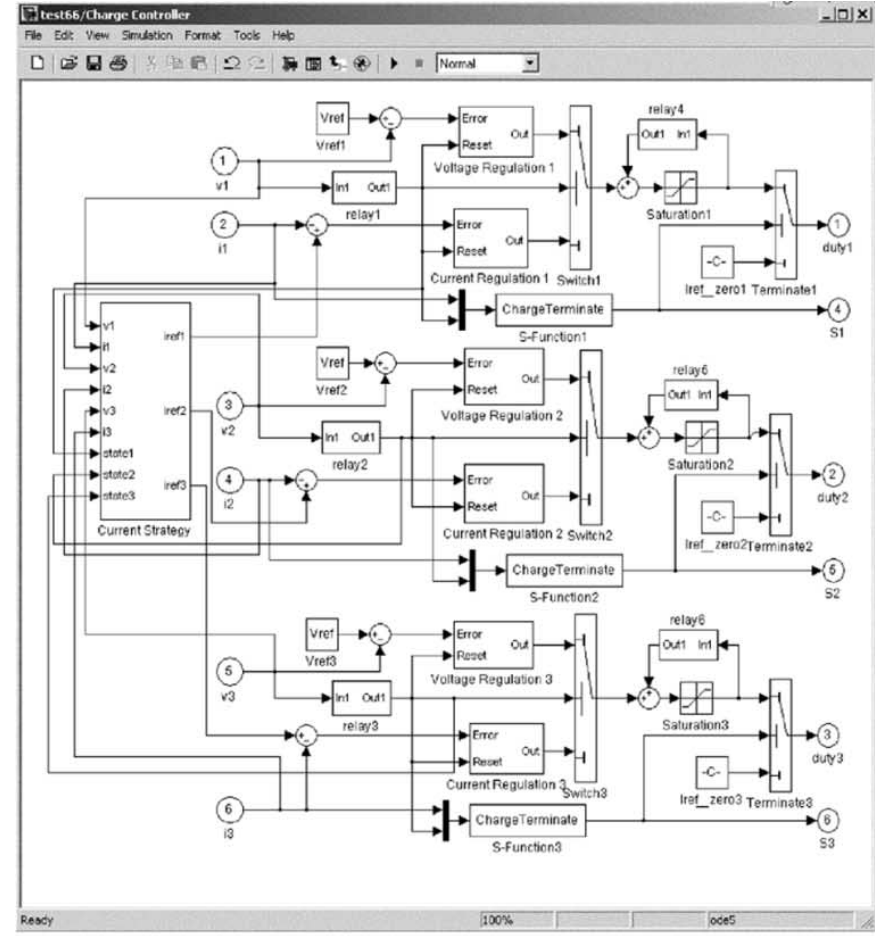

(a)

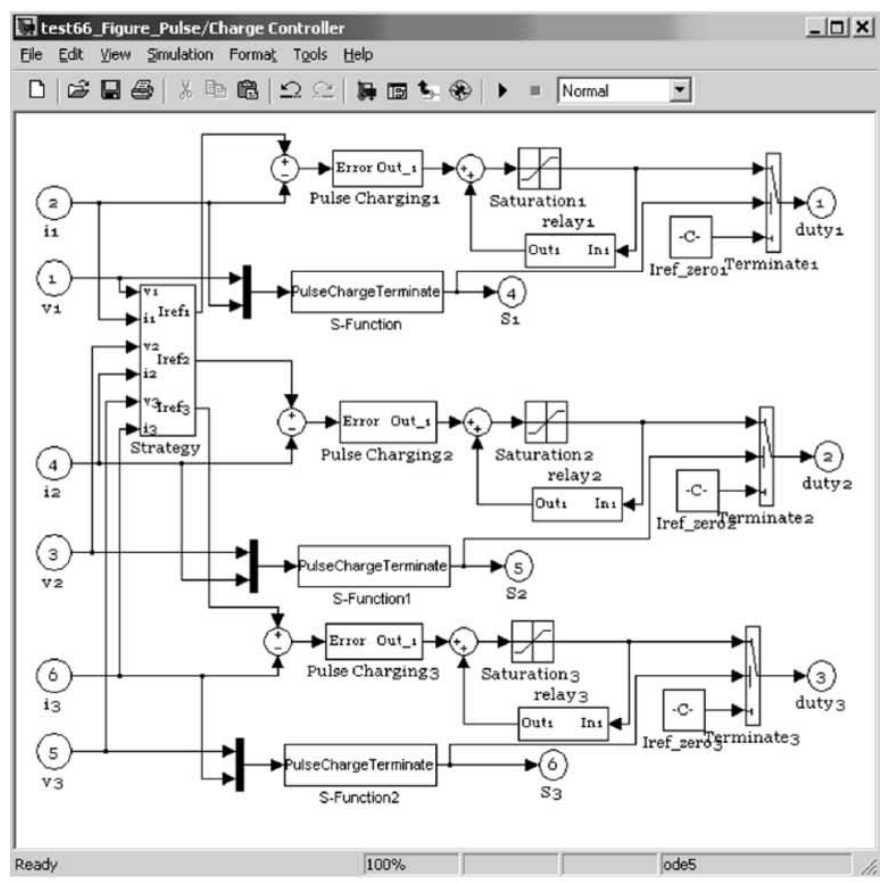

(b)

Fig. 3. Simulink models of the battery charge controllers for (a) dc charging and (b) pulse current charging.

The classical proportional-integral control approach is used to regulate the charging currents and voltages. The current and voltage regulations are formulated in (6) and (7), respectively,

$$
\begin{aligned}
& u=u_{\mathrm{old}}+k_{p i}\left(I_{\text {ref }}-I\right)+k_{i i} \int\left(I_{\text {ref }}-I\right) d t \\
& u=u_{\text {old }}+k_{p v}\left(V_{\text {ref }}-V\right)+k_{i v} \int\left(V_{\text {ref }}-V\right) d t
\end{aligned}
$$




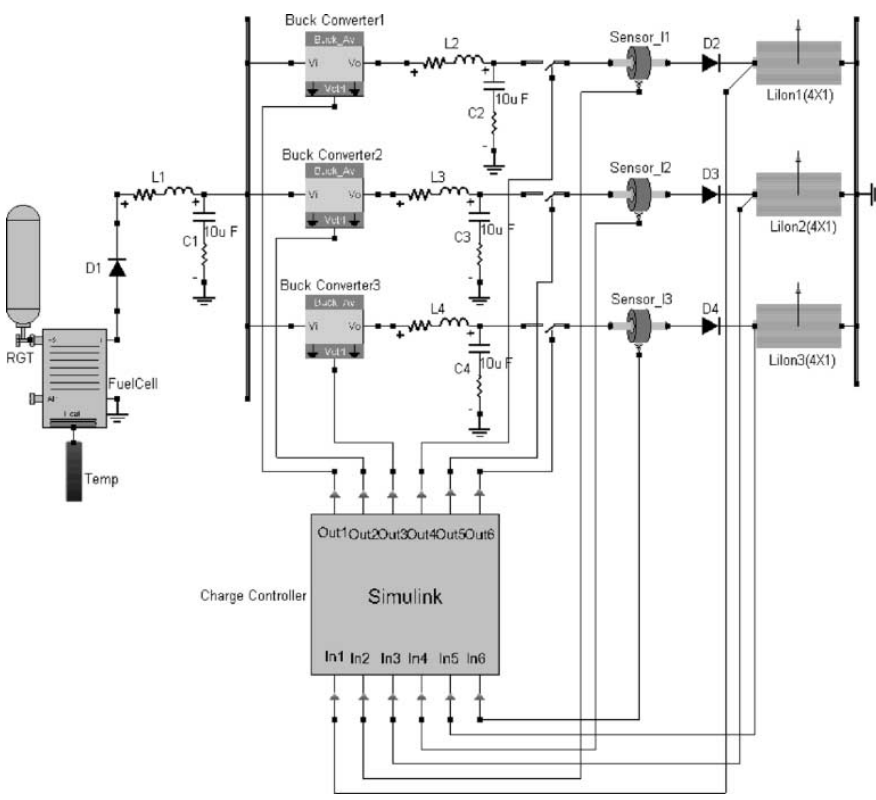

Fig. 4. Schematic view of the fuel-cell-powered battery-charging station.

where $V, I$ are the sampled voltage and current of the battery, $u$ and $u_{\text {old }}$ are the current and previous duty cycles used to control the buck converter, both of which are values between $0.05-0.95, V_{\text {ref }}$ and $I_{\text {ref }}$ are the reference end voltage and reference charging current of the battery, and $k_{p i}, k_{i i}$ and $k_{p v}, k_{i v}$ are proportional and integral gains for current and voltage regulations, respectively.

\section{Simulation Results}

In order to compare the performance of three control strategies for active power sharing, simulation studies were first conducted in the VTB [11]. Fig. 4 shows the VTB schematic view of the system shown in Fig. 1. The power source is a 25-cell PEM fuel-cell stack. Each battery is a $4 \times 1$ (series by parallel connections) array of lithium-ion cells. The capacity of each battery is $1500 \mathrm{mAh}$. The initial states of charge of the batteries are 0.7, 0.5 , and 0.3 , respectively. Each power converter is implemented by a switching-average buck converter model in series with a low-pass filter.

The controller is implemented in the Simulink models as shown in Fig. 3. It is embedded in the VTB through a VTB-Simulink interface and it can co-simulate with the VTB interactively. The charging currents and battery voltages are sensed and fed to the controller. The controller outputs three duty cycles to the power converters and three commands for the switches. The proportional and integral gains for current regulation are 0.05 and 0.002 , respectively. The proportional and integral gains for voltage regulation are 0.01 and 0.001 , respectively. In the studied dc charging strategies, the sum of the total available charging currents is limited to $2 \mathrm{~A}$. While the reference charging currents are set $0.66 \mathrm{~A}$ for all of the batteries under the baseline strategy, they are $0.38,0.66$, and $0.96 \mathrm{~A}$, respectively, under proportional rate charging strategy. The limit of the charging currents is $1.5 \mathrm{~A}$ for pulse current charging strategy taking the safe charging current of the batteries into consideration. The duty cycles of the charging currents are $20 \%, 33.3 \%$, and $46.7 \%$, respectively, for three batteries. The charging process stops when the battery voltage during the low-pulse duration reaches the reference voltage.

This system is simulated with the three different charging strategies for $2 \mathrm{~h}(7200 \mathrm{~s})$. The simulated charging currents and states of charge of the batteries under three charging strategies are shown in Fig. 5(a)-(f), respectively.

Obviously, with the baseline strategy, the state-of-charge of the battery with highest initial voltage increases more rapidly than the others. It is seen from Fig. 5(d) and (f) that, with proportional rate charging strategy and pulse current charging strategy, the state-of-charge of the battery with lowest initial voltage increases more rapidly than the others. Therefore, these two strategies can help reduce the total charging time. From the simulation results, the following conclusions can be drawn for the three control strategies.

1) While the battery of the highest initial state-of-charge becomes full fastest with the baseline charging strategy, the total time to fully charge all the batteries is the longest if there are apparent differences in the initial state-ofcharge. It is seen from Fig. 5(a) that it takes about 4050 and $6900 \mathrm{~s}$ for the first and last batteries, respectively, to become full.

2) Compared with the baseline strategy, the total charging time with proportional rate charging strategy is shorter. It is shown in Fig. 5(c) that it takes about $5900 \mathrm{~s}$ for all of the batteries to become full with proportional rate charging strategy.

3) Among the three control strategies, pulse current charging strategy achieves the shortest total charging time because the individual charging current is relatively large. With pulse current charging strategy, it takes about $5400 \mathrm{~s}$ for all the batteries to become full, $1500 \mathrm{~s}$ shorter than that with the baseline strategy and $500 \mathrm{~s}$ shorter than that with proportional rate charging strategy.

4) It can be seen that all of the batteries can become fully charged almost simultaneously when they are charged with an appropriate set of proportional-rate continuous currents or pulse currents. Therefore, it is true that proportional rate charging strategy and pulse current strategy are effective for charging the batteries.

From the above, we can see that proportional rate charging strategy is superior to the baseline strategy with respect to reducing the total charging time. In order to give a wider view of this advantage, the simulation is run five times each at nine discrete initial states to compare the total charging time with these two strategies under different initial conditions. Fig. 6 shows the plots of the total charging time against the average state-ofcharge of three batteries under equal rate charging strategy and proportional rate charging strategy for different initial battery states. The states of charge of the three batteries have equal difference and the average state-of-charge is their algebraic mean value. It is seen from Fig. 6 that the total charging time decreases with the average state-of-charge in spite of the control strategy or initial states of the batteries. For every initial average state-of-charge, the total charging time with proportional rate 


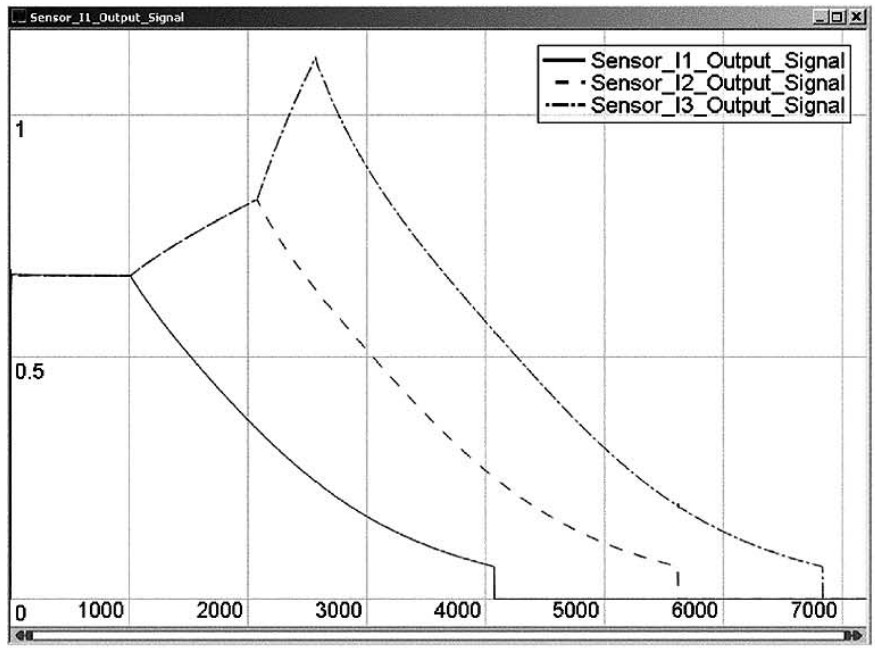

(a) charging currents

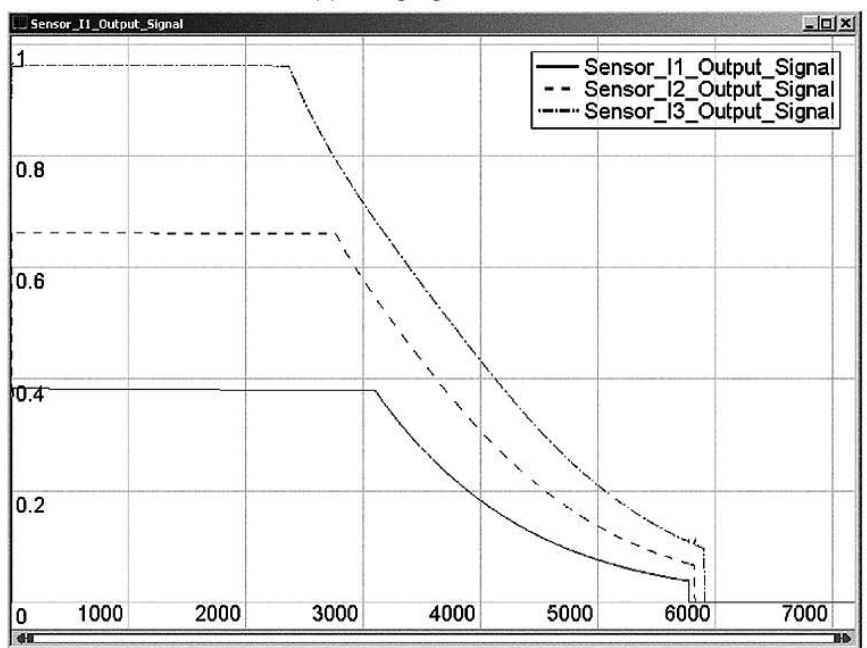

(c) charging currents

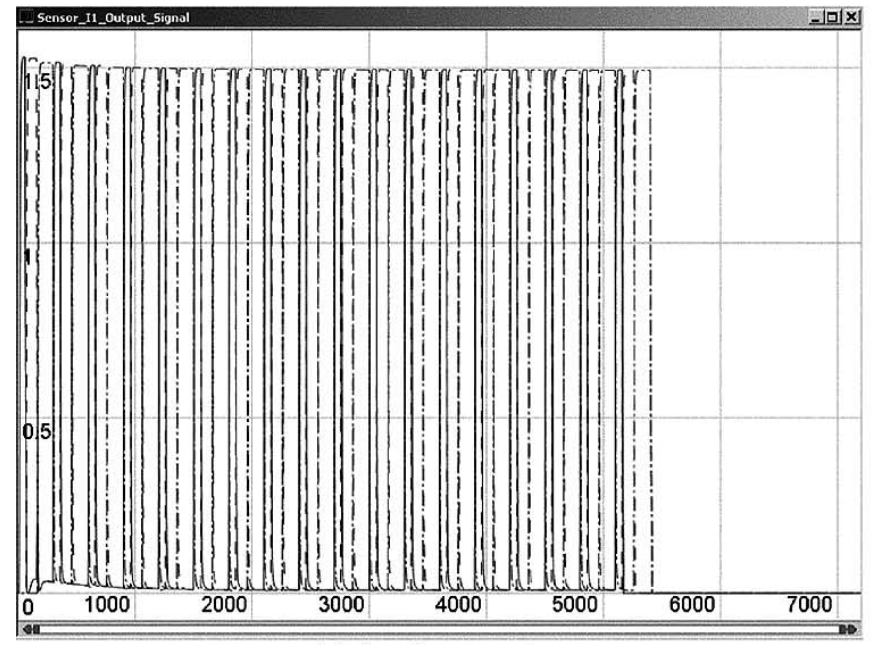

(e) charging currents

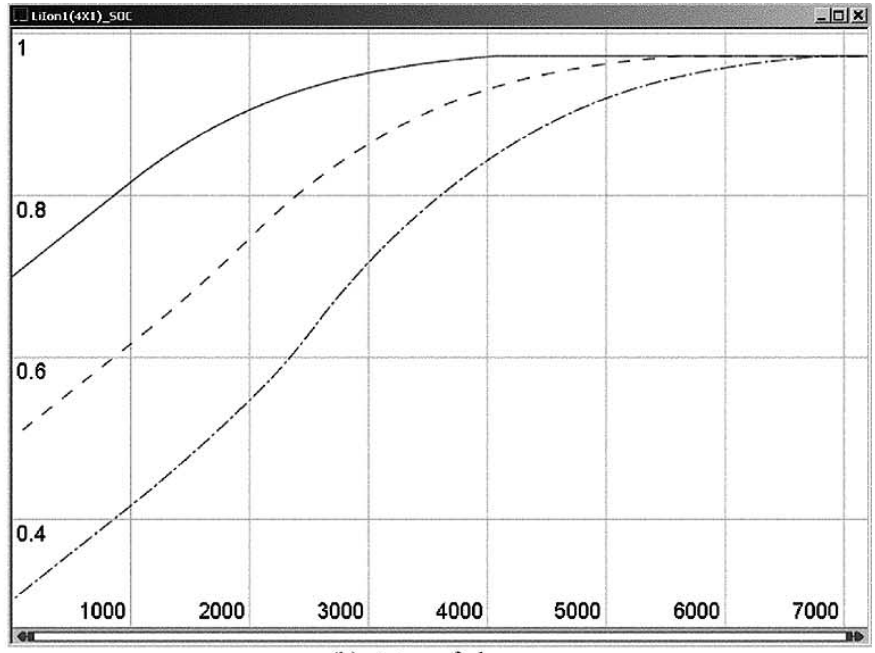

(b) states of charge

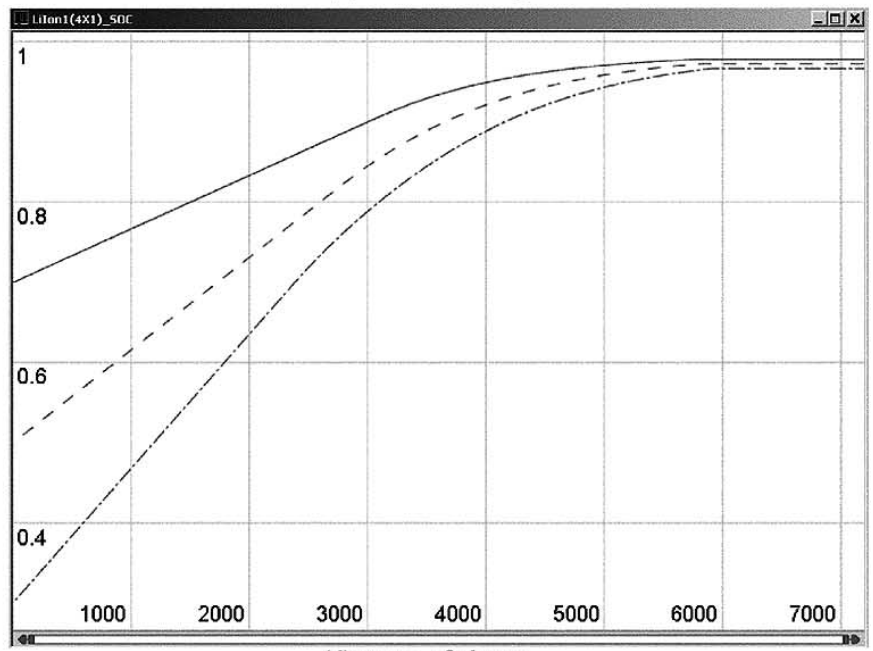

(d) states of charge

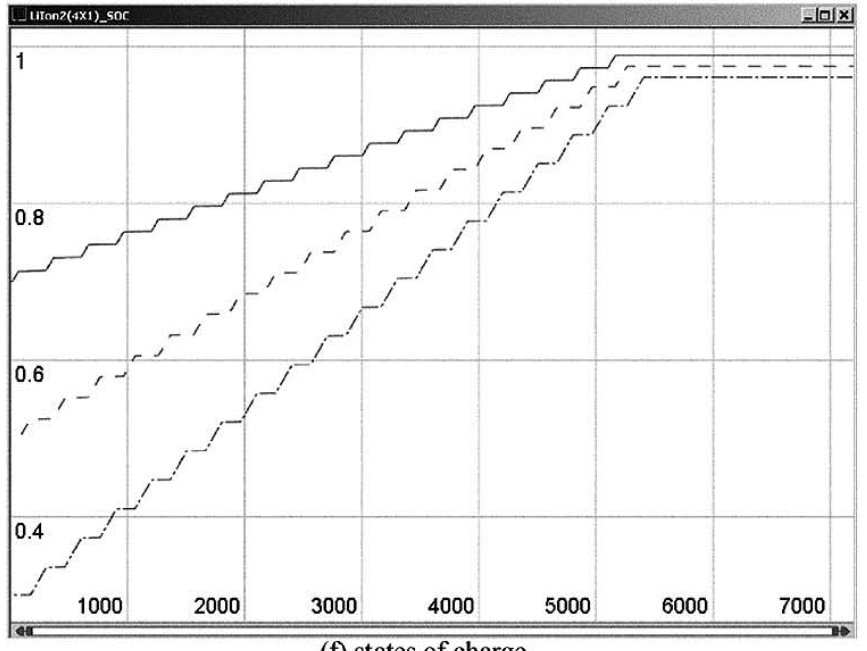

(f) states of charge

Fig. 5. Charging currents and states of charge under three control strategies: (from top to bottom) equal rate charging, proportional rate charging, and pulse current charging.

charging strategy is shorter than that with equal rate charging strategy. The total charging time increases with the difference in the initial state-of-charge with both strategies. The reason for proportional rate charging strategy is that the error of the initial state-of-charge estimation becomes bigger when their differ- ences increase. For equal rate charging strategy, this is because it takes a longer time to charge the most depleted battery when the difference becomes bigger. When all batteries have the identical initial state-of-charge, both strategies have the same effect on the current sharing and the total charging time is identical. 


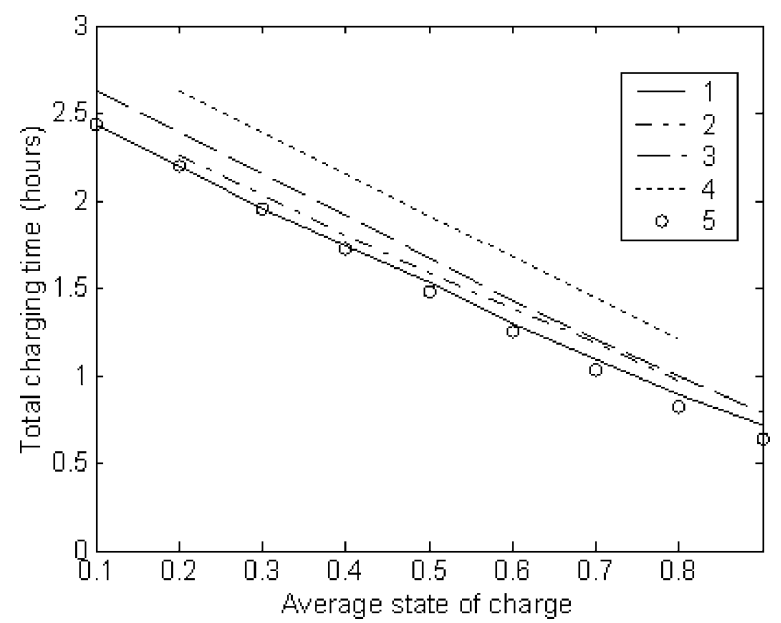

Fig. 6. Plots of the total charging time against the average state-of-charge of three batteries with equal rate-charging strategy and proportional rate-charging strategy under different initial battery states. 1: with proportional rate-charging strategy and a maximum initial state-of-charge difference of $20 \%$; 2: with proportional rate-charging strategy and a maximum initial state-of-charge differennce of $40 \%$; 3 : with equal rate-charging strategy and a maximum initial state-of-charge differennce of 20\%; 4: with equal rate-charging strategy and a maximum initial state-of-charge differennce of $40 \%$; and 5: with either of the charging strategies and the same initial state-of-charge.

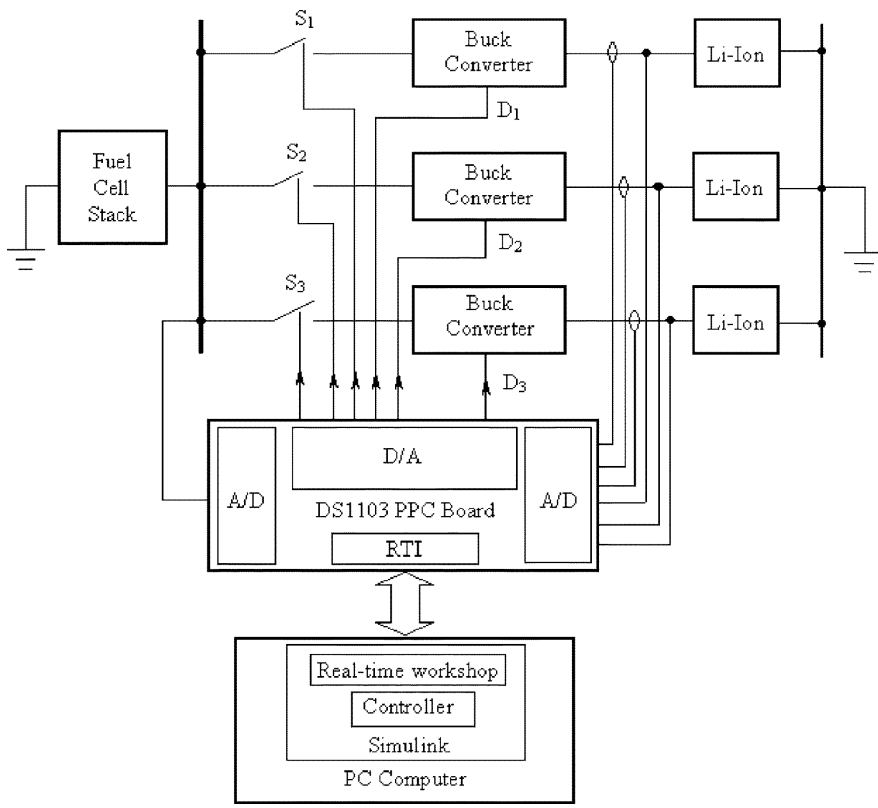

Fig. 7. Block diagram of experiment environment.

\section{EXPERIMENT VALIDATION}

Next, these control strategies were validated with real hardware. A prototype of the fuel-cell-powered battery-charging station was built using an H-Power D35 PEM fuel-cell stack as the power source. This stack had a nominal power capacity of $35 \mathrm{~W}$ and a nominal 24-V open-circuit voltage. Three four-cell Panasonic lithium-ion batteries were used. The nominal capacity of each battery was $1500 \mathrm{mAh}$. Three buck converters were built on one single board to distribute the charging current. The block diagram of the experiment environment is shown in Fig. 7. The charging algorithms implementing the control strategies resided on a general-purpose dSPACE real-time controller board, which also housed the hardware interface consisting of multichannel $\mathrm{A} / \mathrm{D}$ and $\mathrm{D} / \mathrm{A}$ converters. The charging control algorithms were designed and implemented using MATLAB/ Simulink and the codes were then compiled and dropped onto a dSPACE DS1103 PPC controller board to control the real hardware. The charging currents and battery voltages were monitored and input to the dSPACE controller board through the A/D converters mounted on it. The power source bus voltage was also an input variable for monitoring purposes. The real-time controller provided the switch duty commands to each buck converter. The circuit protection function was also implemented within the software.

Tests were conducted using the proportional rate charging strategy. In order to ensure that each charging current would never exceed the safe maximum charging current (that was 800 $\mathrm{mA}$ for the batteries used in the experiment), the total charging current was scaled down by half to $1.0 \mathrm{~A}$. For the convenience of demonstration, three batteries were numbered \#1, \#2, and \#3. The initial states-of-charge of batteries \#1-\#3 were $0.75,0.83$, and 0.92 , respectively. Their initial open- circuit voltages were $15.9,16.1$, and $16.4 \mathrm{~V}$, respectively. The initial state-of-charge of the battery was measured using the following approach. Each battery was discharged to full depletion. A constant current was then applied to charge the battery until it was full and the total charging time was recorded. After fully depleting this battery, charging this battery with the same current for a proportion (equal to the initial state-of-charge in magnitude) of the total charging time could obtain a desired initial state-of- charge.

According to the initial states-of-charge of the batteries, the controller selected the charging currents as $0.5,0.35$, and 0.15 A for batteries \#1-\#3, respectively. The measured battery voltages and charging currents are shown in Fig. 8, where the simulated voltages and currents are also plotted. Small differences in the voltages between simulation results and experiment data were observed and this was due to the fact that more detailed transients were not characterized in the battery model. The ripples in the currents were very small and this was because these measured signals were prefiltered and because current transducers with high resolution were used to measure the charging currents. It can be said that the simulation results matched experiment data very well. From Fig. 8, it is seen that the battery with the lowest initial voltage (and, thus, the least initial charge) was charged with the highest current, and its voltage increased more rapidly than the others. This was also predicted by the simulation. This feature implies that proportional rate charging strategy can help to reduce the total charging time. After a little while charging, the voltages of batteries \#1 and \#2 exceeded the voltage of the third battery. The voltages of these two batteries reached the reference voltage almost simultaneously, and then their currents tapered. When these currents approached $0.15 \mathrm{~A}$, the third battery reached the reference voltage and its current tapered. It is also seen that all three batteries became fully charged almost simultaneously.

\section{CONCLUSION}

This paper has presented an effective system design for a fuel-cell-powered battery-charging station. Three control strategies coordinating the active power sharing among multiple bat- 


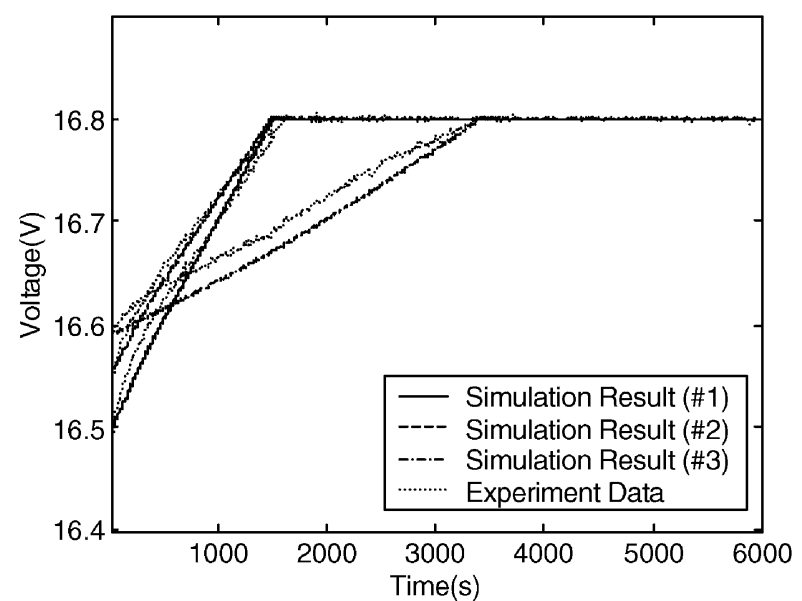

(a) From top to down (on the left vertical axis): voltages of batteries $\# 3$, $\# 2$, and

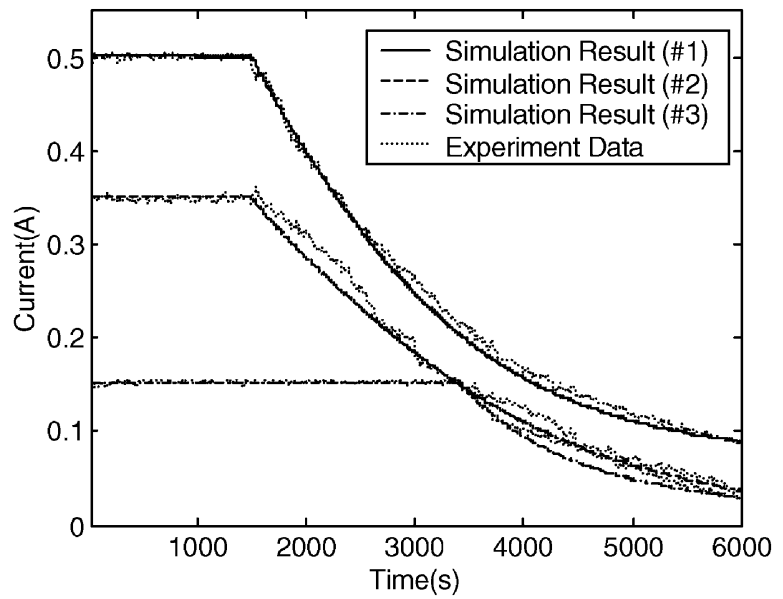

(b) F rom top to down: charging currents for batteries \#1, \#2, and \#3

Fig. 8. Measured battery voltage voltages and charging currents. (a) Voltages. (b) Currents.

tery branches were developed and compared. The Simulink definitions of the battery charging controllers are used for both system simulation and experimental runtime. Simulation results show that, with equal rate charging strategy, the battery with the highest initial state-of-charge becomes full fastest but the total charging time is longest. Proportional rate charging strategy is superior to equal rate charging strategy in respect to the total charging time. The total charging time increases with the difference in the initial state-of-charge of each battery with both strategies. The total charging time is minimum with pulse current charging strategy. It is possible for all the batteries to become full almost simultaneously when they are charged with appropriate proportional constant currents or pulse currents. Experimental tests were performed and the results validated these control strategies and simulation results.

\section{REFERENCES}

[1] R. J. Brodd, "Overview: Rechargeable battery systems," in Proc. WESCON'93, 1993, pp. 206-209.
[2] B. Rohland, J. Nitsch, and H. Wendt, "Hydrogen and fuel cells - The clean energy system," J. Power Sources, vol. 37, no. 1-2, pp. 271-277, Jan. 1992.

[3] A. Heinzel, C. Hebling, M. Müller, M. Zedda, and C. Müller, "Fuel cells for low power applications," J. Power Sources, vol. 105, no. 2, pp. 148-153, Mar. 2002.

[4] Z. Jiang and R. Dougal, "Control design and testing of a novel fuel-cellpowered battery-charging station," in Proc. IEEE APEC, Miami, FL, Feb. 10-14, 2003, pp. 1127-1133.

[5] — , "Design and testing of a fuel-cell-powered battery-charging station," J. Power Sources, vol. 115, no. 2, pp. 279-287, Apr. 2003.

[6] M. M. Bernardi and M. W. Verbrugge, "A mathematical model of the solid-polymer-electrolyte fuel cell," J. Electrochem. Soc., vol. 139, no. 9, pp. 2477-2491, Sept. 1992.

[7] J. Kim, S. M. Lee, and S. Srinivasan, "Modeling of proton exchange membrane fuel cell performance with an empirical equation," J. Electrochem. Soc., vol. 142, no. 8, pp. 2670-2674, Aug. 1995.

[8] L. Song and J. W. Evans, "Electrochemical-thermal model of lithium polymer batteries," J. Electrochem. Soc., vol. 147, no. 6, pp. 2086-2095, 2000 .

[9] J. Li, E. Murphy, J. Winnick, and P. A. Kohl, "The effects of pulse charging on cycling characteristics of commercial lithium-ion batteries," J. Power Sources, vol. 102, no. 1-2, pp. 302-309, Dec. 2001.

[10] Z. Jiang and R. Dougal, "Strategy for active power sharing in a fuel-cellpowered charging station for advanced technology batteries," in Proc. IEEE PESC, vol. 1, Acapulco, Mexico, June 15-19, 2003, pp. 81-87.

[11] T. Lovett, A. Monti, E. Santi, and R. Dougal, "A multilanguage environment for interactive simulation and development of controls for power electronics," in Proc. IEEE PESC, vol. 3, 2001, pp. 1725-1729.

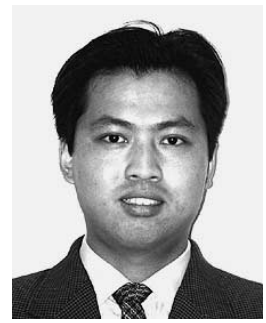

Zhenhua Jiang (S'01-M'04) received the B.Sc. and M.Sc. degrees in electrical engineering from Huazhong University of Science and Technology, Wuhan, China, in 1997 and 2000, respectively, and the Ph.D. degree in electrical engineering from the University of South Carolina, Columbia, in 2003.

$\mathrm{He}$ is currently a Postdoctoral Fellow at the University of South Carolina. His primary research interests include controls, application of power electronics in new energy systems such as electromechanical, photovoltaic, and electrochemical energy conversion systems, battery energy storage systems and hybrid fuel-cell power sources, and modeling and simulation of interdisciplinary systems.

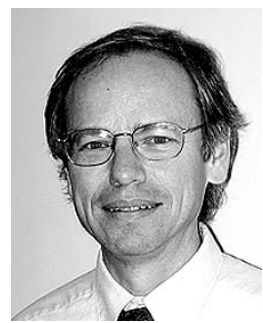

Roger A. Dougal (S'74-M'79-SM'94) received the $\mathrm{Ph} . \mathrm{D}$. degree in electrical engineering from Texas Tech University, Lubbock.

He joined the faculty of the University of South Carolina, Columbia, in 1983. He is Director of the Virtual Test Bed (VTB) project, a multidisciplinary, multi-university effort to develop a comprehensive simulation and virtual prototyping environment for advanced power sources and systems, integrating power electronics, electromechanics, electrochemistry, and controls into a common test bed. The VTB is unique in allowing the simulation of multidisciplinary systems by importing models from discipline-specific source languages to a common workspace. In addition to modeling and simulation, his expertise includes power electronics, physical electronics, and electrochemical power sources.

Dr. Dougal has received the Samuel Litman Distinguished Professor of Engineering Award and has been honored as a Carolina Research Professor. 